\title{
Gauge-invariant gravitational wave modes in pre-big bang cosmology
}

\author{
Valerio Faraoni \\ Physics Department, Bishop's University, 2600 College Street, Sherbrooke, Québec, Canada J1M 1Z7 \\ emailvfaraoni@ubishops.ca
}

Received: date / Revised version: date

\begin{abstract}
The $t<0$ branch of pre-big bang cosmological scenarios is subject to a gravitational wave instability. The unstable behaviour of tensor perturbations is derived in a very simple way in Hwang's covariant and gauge-invariant formalism developed for extended theories of gravity. A simple interpretation of this instability as the effect of an "antifriction" is given, and it is argued that a universe must eventually enter the expanding phase.
\end{abstract}

PACS. 98.80.Bp modified theories of gravity - 98.80.Cq Origin and formation of the Universe - 04.50.Kd particle theories

\section{Introduction}

The application of string theory to cosmology has produced, among other things, pre-big bang cosmology (see [1,2] for a recent exhaustive review). Low-energy string cosmology can be described by the action of the gravitondilaton sector

$$
S=\frac{1}{2 \lambda_{S}^{d-1}} \int d^{d+1} x \sqrt{-g} \mathrm{e}^{-\Phi}\left(R+g^{\mu \nu} \nabla_{\mu} \Phi \nabla_{\nu} \Phi\right)
$$

plus matter, where $\lambda_{S}$ is the natural unit of length, $g_{\mu \nu}$ is the metric tensor with determinant $g$, and $R$ is the Ricci curvature. Two features are characteristic of string theories and unavoidable: the first is the presence of the dilaton field $\Phi$ together with the metric tensor $g_{\mu \nu}$, and the second is the non-minimal coupling of $\Phi$ to the Ricci scalar $R$. Following [1, we neglect for simplicity the matter sector and focus on the cosmological effects of the dilaton. Assuming the extra spatial dimensions to be compactified, the action can be reduced to the Brans-Dicke form

$$
S_{B D}=\int d^{4} x \sqrt{-g}\left(\frac{\varphi R}{2}-\frac{\omega_{0}}{\varphi} g^{\mu \nu} \nabla_{\mu} \varphi \nabla_{\nu} \varphi\right)
$$

provided that $\omega_{0}=-1$ (it is well know that the low-energy limit of the bosonic string, the prototype of string theories, leads to Brans-Dicke gravity with Brans-Dicke parameter $\omega_{0}=-1[3]$ ).

In pre-big bang cosmology it is common to use the shifted dilaton $\bar{\Phi} \equiv \Phi-\ln \left(\Pi_{i} a_{i}\right)$, where the $a_{i}$ are the scale factors of the $d$-dimensional Bianchi metric $d s^{2}=-d t^{2}+$ $\sum_{i} a_{i}^{2}\left(d x^{i}\right)^{2}$. Here we consider spatially flat FriedmannLemaitre-Robertson-Walker (FLRW) metrics, which are the most recurrent in pre-big bang cosmology, and $d=3$, the extra spatial dimensions being compactified, therefore $\bar{\Phi}=\Phi-3 \ln a$, where $a$ is the scale factor of the threedimensional spatial sections and

$$
\varphi=\mathrm{e}^{-\Phi}=\frac{\mathrm{e}^{-\bar{\Phi}}}{a^{3}}
$$

The following solutions derived using scale factor duality transformations 4] are representative of pre-big bang cosmology [1,2]:

$$
\begin{aligned}
& a_{(1)}(t)=t^{1 / \sqrt{3}}, \quad \bar{\Phi}_{(1)}(t)=-\ln t \quad(t>0) \\
& a_{(2)}(t)=(-t)^{1 / \sqrt{3}}, \quad \bar{\Phi}_{(2)}(t)=-\ln (-t) \quad(t<0) \\
& a_{(3)}(t)=t^{-1 / \sqrt{3}}, \quad \bar{\Phi}_{(3)}(t)=-\ln t \quad(t>0) \\
& a_{(4)}(t)=(-t)^{-1 / \sqrt{3}}, \quad \bar{\Phi}_{(4)}(t)=-\ln (-t) \quad(t<0)
\end{aligned}
$$

There is a singularity at $t=0$, which is hoped to be somehow eliminated due to the existence of a fundamental minimum length, the size of the string, so that the two branches of pre- and post-big bang can be joined smoothly through a bounce or a "graceful exit" [2,1].

The stability of these solutions is an issue. Gravitational instabilities are well known to plague pre-big bang cosmology [1] and here we want to study tensor perturbations and their stability. Inhomogeneous perturbations in cosmology are subject to the notorious gauge-dependence problems and need to be analyzed in a gauge-invariant way. In the effective Brans-Dicke theory (2), inhomogeneous perturbations are best analyzed using the covariant 
and gauge-invariant formalism of Bardeen, Ellis and Bruni [5] 6] in the version developed by Hwang and Noh [7] for alternative gravity described by the action

$S=\int d^{4} x \sqrt{-g}\left[\frac{f(\phi, R)}{2}-\frac{\omega(\phi)}{2} g^{\mu \nu} \nabla_{\mu} \phi \nabla_{\nu} \phi-V(\phi)\right]$.

This formalism is particularly convenient to analyze perturbations in the pre-big bang scenarios described by (2). In the next section, the basic variables and equations of this formalism are rewieved and applied to pre-big bang cosmology.

\section{The gauge-invariant formalism}

The metric components are written as

$$
\begin{aligned}
& g_{00}=-a^{2}(1+2 A Y), \\
& g_{0 i}=-a^{2} B Y_{i}, \\
& g_{i j}=a^{2}\left[h_{i j}\left(1+2 H_{L}\right)+2 H_{T} Y_{i j}\right],
\end{aligned}
$$

where $h_{i j}$ is the three-dimensional metric of the FLRW background with associated covariant derivative $\bar{\nabla}_{i}$. The scalar harmonics $Y$ are the eigenfunctions of the eigenvalue problem

$$
\bar{\nabla}_{i} \bar{\nabla}^{i} Y=-k^{2} Y,
$$

with eigenvalue $k$, while the vector and tensor harmonics $Y_{i}$ and $Y_{i j}$ satisfy

$$
Y_{i}=-\frac{1}{k} \bar{\nabla}_{i} Y, \quad Y_{i j}=\frac{1}{k^{2}} \bar{\nabla}_{i} \bar{\nabla}_{j} Y+\frac{1}{3} Y h_{i j} .
$$

The gauge-invariant variables used in Hwang's formalism are the Bardeen [5] potentials

$$
\begin{aligned}
& \Phi_{H}=H_{L}+\frac{H_{T}}{3}+\frac{\dot{a}}{k}\left(B-\frac{a}{k} \dot{H}_{T}\right), \\
& \Phi_{A}=A+\frac{\dot{a}}{k}\left(B-\frac{a}{k} \dot{H}_{T}\right)+\frac{a}{k}\left[\dot{B}-\frac{1}{k}\left(a \dot{H}_{T}\right) \cdot\right]
\end{aligned}
$$

and the Ellis-Bruni [6] variable

$$
\Delta \phi=\delta \phi+\frac{a}{k} \dot{\phi}\left(B-\frac{a}{k} \dot{H}_{T}\right),
$$

where $a$ is the scale factor of the background FLRW line element

$$
d s^{2}=-d t^{2}+a^{2}(t)\left(d x^{2}+d y^{2}+d z^{2}\right)
$$

and an overdot denotes differentiation with respect to the comoving time $t$. The first order equations for the gaugeinvariant perturbations are [7]

$\Delta \ddot{\phi}+\left(3 H+\frac{\dot{\phi}}{\omega} \frac{d \omega}{d \phi}\right) \Delta \dot{\phi}$

$$
\begin{aligned}
& +\left[\frac{k^{2}}{a^{2}}+\frac{\dot{\phi}^{2}}{2} \frac{d}{d \phi}\left(\frac{1}{\omega} \frac{d \omega}{d \phi}\right)-\frac{d}{d \phi}\left(\frac{1}{2 \omega} \frac{\partial f}{\partial \phi}-\frac{1}{\omega} \frac{d V}{d \phi}\right)\right] \Delta \phi \\
& =\dot{\phi}\left(\dot{\Phi}_{A}-3 \dot{\Phi}_{H}\right)+\frac{\Phi_{A}}{\omega}\left(\frac{\partial f}{\partial \phi}-2 \frac{d V}{d \phi}\right) \\
& +\frac{1}{2 \omega} \frac{\partial^{2} f}{\partial \phi \partial R} \Delta R
\end{aligned}
$$

$$
\begin{aligned}
& \Delta \ddot{F}+ 3 H \Delta \dot{F}+\left(\frac{k^{2}}{a^{2}}-\frac{R}{3}\right) \Delta F+\frac{F}{3} \Delta R+\frac{2}{3} \omega \dot{\phi} \Delta \dot{\phi} \\
&+ \frac{1}{3}\left(\dot{\phi}^{2} \frac{d \omega}{d \phi}+2 \frac{\partial f}{\partial \phi}-4 \frac{d V}{d \phi}\right) \Delta \phi \\
&= \dot{F}\left(\dot{\Phi}_{A}-3 \dot{\Phi}_{H}\right)+\frac{2}{3}(F R-2 f+4 V) \Phi_{A},(19) \\
& \ddot{H}_{T}+\left(3 H+\frac{\dot{F}}{F}\right) \dot{H}_{T}+\frac{k^{2}}{a^{2}} H_{T}=0
\end{aligned}
$$

$-\dot{\Phi}_{H}+\left(H+\frac{\dot{F}}{2 F}\right) \Phi_{A}=\frac{1}{2}\left(\frac{\Delta \dot{F}}{F}-H \frac{\Delta F}{F}+\frac{\omega}{F} \dot{\phi} \Delta \phi\right)$,$$
\left(\frac{k}{a}\right)^{2} \Phi_{H}+\frac{1}{2}\left(\frac{\omega}{F} \dot{\phi}^{2}+\frac{3}{2} \frac{\dot{F}^{2}}{F^{2}}\right) \Phi_{A}
$$$$
=\frac{1}{2}\left\{\frac{3}{2} \frac{\dot{F} \Delta \dot{F}}{F^{2}}+\left(3 \dot{H}-\frac{k^{2}}{a^{2}}-\frac{3 H}{2} \frac{\dot{F}}{F}\right) \frac{\Delta F}{F}+\frac{\omega}{F} \dot{\phi} \Delta \dot{\phi}\right.
$$$$
\left.+\frac{1}{2 F}\left[\dot{\phi}^{2} \frac{d \omega}{d \phi}-\frac{\partial f}{\partial \phi}+2 \frac{d V}{d \phi}+6 \omega \dot{\phi}\left(H+\frac{\dot{F}}{2 F}\right)\right] \Delta \phi\right\},
$$

$$
\Phi_{A}+\Phi_{H}=-\frac{\Delta F}{F},
$$

$$
\begin{aligned}
\ddot{\Phi}_{H} & +H \dot{\Phi}_{H}+\left(H+\frac{\dot{F}}{2 F}\right)\left(2 \dot{\Phi}_{H}-\dot{\Phi}_{A}\right) \\
& +\frac{1}{2 F}(f-2 V-R F) \Phi_{A} \\
& =-\frac{1}{2}\left[\frac{\Delta \ddot{F}}{F}+2 H \frac{\Delta \dot{F}}{F}+(P-\rho) \frac{\Delta F}{2 F}+\frac{\omega}{F} \dot{\phi} \Delta \dot{\phi}\right. \\
& \left.+\frac{1}{2 F}\left(\dot{\phi}^{2} \frac{d \omega}{d \phi}+\frac{\partial f}{\partial \phi}-2 \frac{d V}{d \phi}\right) \Delta \phi\right],
\end{aligned}
$$

$$
\begin{aligned}
\Delta R & =6\left[\ddot{\Phi}_{H}+4 H \dot{\Phi}_{H}+\frac{2}{3} \frac{k^{2}}{a^{2}} \Phi_{H}-H \dot{\Phi}_{A}\right. \\
& \left.-\left(2 \dot{H}+4 H^{2}-\frac{k^{2}}{3 a^{2}}\right) \Phi_{A}\right],
\end{aligned}
$$


where $F \equiv \partial f / \partial R$ and $F_{c} \equiv h_{c}^{d} \nabla_{d} F$ is the spatial projection of the gradient of $F$ and the effective energy density and pressure of the scalar are

$$
\begin{aligned}
& \rho=\frac{1}{F}\left[\frac{\omega \dot{\phi}^{2}}{2}+\frac{(R F-f+2 V)}{2}-3 H \dot{F}+\nabla^{c} F_{c}\right], \\
& P=\frac{1}{F}\left[\frac{\omega \dot{\phi}^{2}}{2}+\frac{(f-R F-2 V)}{2}+\ddot{F}+2 H \dot{F}-\frac{2}{3} \nabla^{c} F_{c}\right] .
\end{aligned}
$$

While the analytic solution of the perturbation equations in a background other than de Sitter 8 is a daunting task, eq. (20) for the tensor modes $H_{T}$ is particularly simple since it is decoupled from the equations for the other modes. In the zero-momentum limit $k \rightarrow 0$ it reduces to

$$
\ddot{H}_{T}+\left(3 H+\frac{\dot{\varphi}}{\varphi}\right) \dot{H}_{T}=0
$$

using the fact that $F=\varphi$. Note also that, using the well known result of pre-big bang cosmology $\dot{\bar{\Phi}}= \pm \sqrt{3} H$ (see, e.g., p. 139 of [1]), the coefficient of $\dot{H}_{T}$ in eq. (20) is $(3 H+\dot{\varphi} / \varphi)=-\dot{\bar{\Phi}}$ and corresponds to a friction term if $\dot{\bar{\Phi}}<0$ and to antifriction if $\dot{\bar{\Phi}}>0$. Positive friction will damp the tensor perturbations, while antifriction enhances them. Eq. (28) yields

$$
\dot{H}_{T}=\frac{C}{a^{3} \varphi},
$$

where $C$ is an integration constant.

Let us consider the contracting pre-big bang solution $a_{(2)}(t)=(-t)^{1 / \sqrt{3}}, \bar{\Phi}_{(2)}(t)=-\ln (-t)$ for $t<0$ : here $\dot{\bar{\Phi}}=-1 / t>0$ and

$$
\dot{H}_{T}=\frac{C}{a^{3} \varphi}=C \mathrm{e}^{\bar{\Phi}}=\frac{C}{-t}
$$

diverges as $t \rightarrow 0^{-}$(for general non-zero values of the arbitrary integration constant $C$ ). Similarly, for the polelike expanding pre-big bang branch $a_{(4)}(t)=(-t)^{-1 / \sqrt{3}}$, $\bar{\Phi}_{(4)}=-\ln (-t)$ (with $\left.t<0\right)$, the tensor perturbations diverge as $t \rightarrow 0^{-}$. For both of these solutions $\dot{\bar{\Phi}}=-1 / t>$ 0 corresponds to antifriction.

Let us consider now the post-big bang $(t>0)$ branch $a_{(1)}(t)=t^{1 / \sqrt{3}}, \bar{\Phi}_{(1)}=-\ln t$; here

$$
\dot{H}_{T}=C \mathrm{e}^{\bar{\Phi}}=\frac{C}{t}
$$

tends to zero as $t \rightarrow+\infty$ irrespective of the value of $C$ and $H_{T}$ becomes constant, corresponding to neutral stability. Note that in this case $\dot{\bar{\Phi}}=-1 / t<0$ corresponds to (positive) friction. The same considerations apply to $a_{(3)}(t)=t^{-1 / \sqrt{3}}, \bar{\Phi}_{(3)}=-\ln t($ for $t>0)$.

\section{Discussion}

The pre-big bang branch is unstable with respect to long wavelength tensor perturbations. This fact is well known, but it appears in a particularly simple way in Hwang's formalism and the discussion is not tied to a particular gauge, as is often the case in the literature. Moreover, in the previous discussion, the instability receives a particularly simple interpretation as the effect of an antifriction in the evolution equation for these gauge-invariant modes, which is inherently due to the fact that the universe contracts. This instability could be regarded as a shortcoming of pre-big bang scenarios. However, it is also possible that breaking the evolution of the pre-big bang branch by means of tensor perturbations (of classical or quantum origin) that grow and drive the universe away for the background evolution helps in solving the long-standing graceful exit problem of joining the pre- and the postbig bang branches. This possibility deserves attention in the future. In any case, it seems that a contracting phase cannot last, eventually becoming unphysical, and that the universe is bound to evolve into an expanding one.

Another aspect of the gravitational wave instability is that, since long wavelength modes can grow, if they are somehow stopped before causing the dynamics to deviate drastically from a FLRW one (which can only be assessed in a more detailed scenario and ideally requires to be confirmed by a second order perturbation analysis), largescale perturbations in the cosmic microwave background would be induced. While the experimental error in the observations is necessarily large at these large scales, there is hope that a signature of pre-big bang physics is still left in the cosmic microwave background. Although stabilization seems a problem at present, the possibility of such a signature makes it worth to pursue this possibility. It is not excluded that, in this case, the growing $H_{T}$ modes induce effects detectable already by the PLANCK satellite 9 . More detailed pre-big bang scenarios stabilized by extra ingredients will be analyzed (also at smaller scales) in future work with this possibility in mind.

\section{Acknowledgments}

We thank a referee for useful comments. This work is supported by the Natural Sciences and Engineering Research Council of Canada (NSERC).

\section{References}

1. M. Gasperini, Elements of String Cosmology (Cambridge University Press, Cambridge, 2007).

2. M. Gasperini, The Universe Before the Big Bang: Cosmology and String Theory (Springer, Berlin, 2008); M. Gasperini and G. Veneziano, Phys. Rept. 373, 1 (2003).

3. C.G. Callan, D. Friedan, E.J. Martinec and M.J. Perry, Nucl. Phys. B 262, 593 (1985); E.S. Fradkin and A.A. Tseytlin, Nucl. Phys. B 261, 1 (1985). 
4. A.A. Tseytlin, Mod. Phys. Lett. A 6, 1721 (1991); G. Veneziano, Phys. Lett. B 265, 287 (1991).

5. J.M. Bardeen 1980, Phys. Rev. D 22, 1882.

6. G.F.R. Ellis and M. Bruni, Phys. Rev. D 40, 1804 (1989); G.F.R. Ellis, J.-C. Hwang and M. Bruni, Phys. Rev. D 401819 (1989); G.F.R. Ellis, M. Bruni and J.-C. Hwang, Phys. Rev. D 42, 1035 (1990); J.-C. Hwang and E.T. Vishniac, Astrophys. J. 353, 1 (1990).

7. J.-C. Hwang, Class. Quantum Grav. 7, 1613 (1990); J.-C. Hwang, Phys. Rev. D 42, 2601 (1990); J.-C. Hwang, Class. Quantum Grav. 8, 195 (1990); J.-C. Hwang, Phys. Rev. D 53, 762 (1996); J.-C. Hwang, Class. Quantum Grav. 14, 1981 (1997); J.-C. Hwang and H. Noh, Phys. Rev. D 54, 1460 (1996); H. Noh and J-C. Hwang, Phys. Lett. B 515, 231 (2001).

8. V. Faraoni, Phys. Rev. D 70, 044037 (2004); Phys. Rev. D 70, 081501(R) (2004); Phys. Rev. D 72, 061501(R) (2005); G. Cognola, E. Elizalde, S. Nojiri, S.D. Odintsov, and S. Zerbini, J. Cosmol. Astropart. Phys. 0502:010 (2005); V. Faraoni and S. Nadeau, Phys. Rev. D 72, 124005 (2005); G. Cognola and S. Zerbini, J. Phys. A 39, 6245 (2006); V. Faraoni and M.N. Jensen, Class. Quantum Grav. 23, 3005 (2006); V. Faraoni, Phys. Rev. D 75, 067302 (2007); G. Cognola and S. Zerbini, arXiv:0802.3967, G. Cognola, M. Gastaldi, and S. Zerbini, Int. J. Theor. Phys. 47, 898 (2008). See also T.P. Sotiriou and V. Faraoni, Rev. Mod. Phys. 82, 451 (2010).

9. PLANCK homepage http://www.rssd.esa.int/index.php?project=Planck 